2 Jacob HS, Goldstein IM, Shapiro I, Craddock PR, Hammerschmidt DE, Weissmann G. Sudden blindness in acute pancreatitis. Possible role of complement-induced retinal leukoembolization. Arch Intern Med 1981; 141(1): 134-136.

3 Agrawal A, McKibbin MA. Purtscher's and Purtscher-like retinopathies: a review. Surv Ophthalmol 2006; 51(2): 129-136.

BLW Nesmith ${ }^{1}$, MS Bitar ${ }^{2}$ and S Schaal ${ }^{1}$

${ }^{1}$ Department of Ophthalmology and Visual Sciences, University of Louisville, Louisville, KY, USA

${ }^{2}$ Department of Ophthalmology and Visual Sciences, University of Illinois at Chicago, Chicago, IL, USA

E-mail: s.schaal@louisville.edu

Eye (2014) 28, 1038-1040; doi:10.1038/eye.2014.85; published online 2 May 2014

\section{Sir,
Response to Nesmith et al}

We thank Nesmith et $a l^{1}$ for their interesting report of anatomical and functional benefit of intravitreal bevacizumab in the treatment of macular edema in a probable case of Purtscher-like retinopathy. The case reported respects the majority of the diagnostic criteria of Purtscher or Purtscher-like retinopathies suggested by Agrawal $^{2}$ and adapted by us, ${ }^{3}$ namely:

- Retinal hemorrhages, in the posterior pole, in small number (1-10).

- Cotton wool spots restricted to the posterior pole.

- Probable or plausible explanatory etiology (chronic pancreatitis, hepatitis C, cirrhosis, and typical presentation).

- Compatible complementary investigation (characteristic fundoscopy, OCT revealing macular edema, and increased C5a).

- The presence of Purtscher flecken ${ }^{4}$ is another diagnostic criterion (in this case there were none visible). Purtscher flecken are considered pathognomonic ${ }^{4}$ and occur in $63 \%$ of Purtscher and Purtscher-like retinopathies $(\mathrm{PuR}){ }^{3}$

Nesmith et al report the first case of PuR with improvement of visual acuity, fundoscopic signs, and macular edema after the injection of intravitreal bevacizumab. Anti-VEGF agents may be of value considering PuR's proposed pathological mechanisms, namely in endothelial dysregulation ${ }^{5}$ and in precapillary arterioles' occlusion with altered retinal microvascular permeability. ${ }^{6}$

However, there must be caution before concluding that bevacizumab is useful in PuR because spontaneous improvement is frequent. In a systematic review regarding Purtscher and Purtscher-like retinopathies, ${ }^{3}$ we identified improvement of visual acuity in PuR patients without treatment (there was no statistically significant difference between visual acuity improvement in patients receiving corticosteroids vs no corticosteroids), as well as improvement in OCT findings in $63 \%$ of the patients, as normalization of the fundoscopic signs in $40 \%$ of the patients with PuR. Observation and treatment of the underlying etiology may continue to be the most reasonable therapeutic option at the moment.

In conclusion, further studies should be performed to ascertain whether there is real benefit in performing a treatment with intravitreal bevacizumab. In fact, an international multicentered trial (given the low incidence of this pathology $\mathrm{y}^{7}$ ) with different treatment arm groups should be initiated, comparing sham injection, intravitreal bevacizumab injection, corticosteroid use, and observation alone.

\section{Conflict of interest}

The authors declare no conflict of interest.

\section{References}

1 Nesmith BLW, Bitar MS, Schaal S. The anatomical and functional benefit of bevacizumab in the treatment of macular edema associated with Purtscher-like retinopathy. Eye (Lond) 2014; 28(8): 1038-1040.

2 Agrawal A, McKibbin M. Purtscher's retinopathies: a review. Surv Ophthalmol 2006; 51: 129-136.

3 Miguel AI, Henriques F, Azevedo LF, Loureiro AJ, Maberley DA. Systematic review of Purtscher's and Purtscher-like retinopathies. Eye (Lond) 2013; 27(1): 1-13.

4 Proença Pina J, Ssi-Yan-Kai K, de Monchy I, Charpentier B, Offret H, Labetoulle M. Purtscher-like retinopathy: case report and review of the literature. J Fr Ophtalmol 2008; 31(6): 609.

5 Harrison TJ, Abbasi CO, Khraishi TA. Purtscher retinopathy: an alternative etiology supported by computer fluid dynamic simulations. Invest Ophthalmol Vis Sci 2011; 52(11): 8102-8107.

6 Medeiros HA, Medeiros JA, Caliari LC, Silva J. Purtscher's and Purtscher-like retinopathies. Rev Bras Oftalmol 2009; 68(2): 114-119.

7 Agrawal A, McKibbin M. Purtscher's retinopathy: epidemiology, clinical features and outcome. Br J Ophthalmol 2007; 91(11): 1456-1459.

AIM Miguel ${ }^{1,2}$, F Henriques ${ }^{1}$, LFR Azevedo², AJR Loureiro ${ }^{1}$ and DAL Maberley ${ }^{3}$

${ }^{1}$ Ophthalmology Department, Central University Hospital of Coimbra, Coimbra, Portugal

${ }^{2}$ Center for Research in Health Technologies and Information Systems (CINTESIS), Faculty of Medicine, University of Porto, Porto, Portugal

${ }^{3}$ Retinal Division, Department of Ophthalmology and Visual Sciences, University of British Columbia, Vancouver, BC, Canada

E-mail: myworld_ana@hotmail.com

Eye (2014) 28, 1040; doi:10.1038/eye.2014.92; published online 2 May 2014 